\title{
STABLE TAXATION SCHEMES IN REGIONAL ENVIRONMENTAL MANAGEMENT
}

\author{
S. Rinaldi*, R. Soncini-Sessa*, \\ and A.B. Whinston** \\ RR-77-10 \\ May 1977
}

*Centro Teoria dei Sistemi, CNR, Milan, Italy.

**H.C. Krannert Graduate School of Industrial Administration, Purdue University, Lafeyette, Indiana, USA.

This work has been supported in part by the Centro Teoria dei Sistemi, CNR, Via Ponzio 34/5, Milan, Italy.

Research Reports provide the formal record of research conducted by the International Institute for Applied Systems Analysis. They are carefully reviewed before publication and represent, in the Institute's best judgment, competent scientific work. Views or opinions expressed herein, however, do not necessarily reflect those of the National Member Organizations supporting the Institute or of the Institute itself. 


\section{PREFACE}

This report is one of a series describing IIASA research into methods for comparing alternative models that could be applied for the establishment of control policies to meet water quality standards. In addition to model evaluation, this project has focused on problems of optimization and conflict resolution in large river basins. 


\begin{abstract}
Economists have frequently proposed the use of taxes to internalize externalities, the unit tax on a particular activity being equal to the marginal social damage it generates. This paper introduces such a taxation scheme, comprising a set of rules which, given a set of polluters, their profits and costs, and a Central Authority for environmental control, generates a set of taxes to be levied on the waste emissions. Different attributes of the taxation schemes are then presented, the most important of which (stability) refers to the possibility of overall eooperation between all the polluters. The paper concludes by examining the implications of the existence of stable taxation schemes and of the imposition of constraints either on the emissions or on the percentage of load removed.
\end{abstract}


1 


\section{Stable Taxation Schemes in Regional}

Environmental Management

\section{INTRODUCTION}

Many opportunities and problems faced by individual decision units can be better dealt with or exploited by group behavior. One example that this paper is concerned with is the problem of dealing with water pollution, and the opportunity presented is for a joint arrangement to treat and transport wastes. Regional or areawide wastewater treatment systems offer economic and environmental benefits to wastewater dischargers. Economic benefits arise from economies of scale and the opportunity to develop a comprehensive, consistent wastewater treatment system which minimizes redundant capacities. Environmental benefits arise from the increased reliability of larger, better funded systems and the opportunity to move effluents to discharge points with minimal adverse impact.

Along with the potential benefits come the problems of how to organize the regional system efficiently including an agreement on how the benefits should be distributed. One mechanism for allocating benefits is through a pricing structure based on the services of a regional authority. Depending on the particular pricing structure and the administrative control exercised by a central coordinating agency, different distributions of profits and benefits result. Certain pricing rules may unduly favor one class of users over another.

A central problem for the investigation is the question of what allocation of benefits among the participants reinforces group adhesion. We refer to a pricing rule as stable if once stated there are no incentives for the users of the service to reject the regional plan. Such an incentive to reject would exist if a smaller group of participants could conceive of a plan 
that would allow for greater rewards. The desirability of central treatment of water depends on the existence of economies of scale. Given that there are economies of scale over the relevant range of demand, the potential for a profitable collaboration among users exists. The options for choosing stable prices depends on the degree of economies of scale.

In presenting the model of a regional system, we consider two possible institutional arrangements. Either the waste treatment plants could be owned and operated by the producer, or they could be operated by a regional authority. The general setting involves a transfer from the producers in the region and the regional authority. The transfer consists of waste discharge, possibly treated, and funds from the producer to the regional authority. The amount of waste discharge to be transferred to the regional authority and the decision on any further treatment is based on achieving the greatest regional profit, where profit is measured by private gains by firms in terms of their own outputs, and both the environmental damages and treatment costs. It should be emphasized that a best regional plan does not necessarily imply one central treatment plant but may consist of a completely decentralized system of treatment plants, one for each discharger. The main point is that the plan developed should take into account the total costs and profits for the entire region. Associated with a regional solution will be a set of charges paid by the firms to the regional system. The charges are both for the treatment of waste that the regional authority carries out (if any) and for the environmental damages. The charges have a variety of purposes; first, they are incentives to the firms to develop production processes which generate less waste discharge; secondly, they are used by the regional authority to pay for the costs of disposing of the waste including compensation for environmental damages; and last, the charges should support the regional system.

In order to understand and analyze the problem of supporting financially a regional system based on overall planning, we will use some of the concepts from the theory of games, and in particular the characteristic function. In order to explain the meaning 
and use of this function, consider a regional system consisting of $\mathrm{N}$ firms plus the regional authority. One way of describing a game is to determine what each collection or coalition of players can achieve. The characteristic function of a coalition is such a measure, for it gives to each coalition what that coalition's total profit is worth. For the regional problem, the characteristic function determines the maximum net profit attainable for any group of firms and the regional authority. In order to specify the function, we must define what the legal conditions governing the formation of a regional system are. For example, without any legal restrictions on direct discharge by a producer, there would be no rationale for any regional system. The legal imposition of restrictions on effluent discharge creates a possible need on the part of the dischargers for some coordination of waste treatment in order to achieve economies of scale. From the viewpoint of the regional authority, it must receive some guidance from the governing political unit on how discharges should be determined. Thus the method for calculating the values of the characteristic function must reflect the legal restrictions on the choices available to the participants.

We shall assume that the regional authority has been given legal responsibility for all discharges. Based on a damage function, it must make appropriate payments for compensation. Dischargers must obtain an agreement with the regional authority for a certain discharge level. Without an agreement, no discharge is possible. Reflecting these conditions, the characteristic function is defined to have the following properties. First, the value of a coalition without the regional authority as a member is zero, since we assume that some waste discharge is generated at any level of production and discharge rights must be granted by the regional authority. Secondly, a coalition with only the Central Agency as a member will have zero value since there is no discharge taking place. Thus the only coalitions with potentially positive value for the characteristic function are those that contain both the regional authority and at least one producer. 
In summary, the characteristic function $\mathrm{V}$ is defined with respect to different collections of dischargers and the regional authority. The value of the function gives the total potential srofit available including taking account of treatment costs and environmental damage. Because of the legal restrictions, zero value is given to coalitions that do not include the regional authority.

Changes in the characteristic function $\mathrm{V}$ indicate how profit lepends on the coalition structure. Thus consider $y_{1}$ and $y_{2}$ listinct collections of all parties $\bar{N}$ (dischargers and central tuthority) where the Central Authority is a member of, say, $y_{1}$. Then $\mathrm{V}\left(y_{2}\right)=0$ and $\mathrm{V}\left(y_{1}\right) \geq 0$. Suppose $\mathrm{V}\left(y_{1}\right) \leq \mathrm{V}\left(y_{1} \cup y_{2}\right)$. In that case, the addition of the members of $y_{2}$ to the group consisting of $y_{1}$ improves the total profit. In the case we are nodelling, a regional authority controlling pollution discharge, this would mean that the increase in environmental damage and treatment costs is less than the additional profit made by $y_{2}$. The reverse condition, $\mathrm{V}\left(y_{1}\right)>\mathrm{V}\left(y_{1} \cup y_{2}\right)$, reflects a situation where the additional members from $y_{2}$ lower the total profits. Inder this circumstance, the environmental damages and treatment costs are extremely high. One would expect strong resistance to having the additional members added to the region.

A major issue explored in the paper is the existence of charges on the dischargers which allows the regional authority to at least cover its costs while at the same time, there is no incentive for any group of dischargers to withdraw from the rejional system. In our formulation of the problem, the regional authority has certain extraordinary power in that a discharger sannot operate without securing some agreement with the authority. Under this assumption, as already mentioned, a virtual veto power is possessed by the regional authority. As long as certain conditions on the profit functions for the firms are satisfied, then ylobally stable arrangements for charging the dischargers exist as long as $\mathrm{V}(\bar{N}) \geq \mathrm{V}(y)$ for all coalitions $y$. In fact, if globally stable charges do exist, the value of the grand coalition must have this property. In general terms, the satisfaction of this 
condition means that overall cooperation is profitable. From a total profit point of view, joint cooperation in regional planning among all dischargers is better than having any smaller group operating in the river basin. Thus, what is best from the optimality point of view can also be achieved via a globally stable pricing system.

The benefits accruing to each party are the net profits after a taxation charge has been levied by the Central Authority. A taxation scheme is acceptable if each coalition member will get a non-negative benefit; it is efficient if the total benefits of any coalition sum to its characteristic function value. Taxation schemes that are both stable and efficient will necessarily be acceptable. Examples that have the above properties and will be described in subsequent sections include: equal amount of benefit per polluter, lexicographic, generalized lexicographic, and a method based on the shapley value.

The benefit accruing to the Central Authority is the summed charges over polluters minus the costs for environmental damage (and in some cases, treatment costs). Environmental damage costs are convex in the effluents; that is, they satisfy the congestion effect. Thus, the existence of these costs represents a counterbalancing force to the formation of the grand coalition $\bar{N}$. If these costs did not occur, then there would exist stable and efficient taxation schemes, and it would be possible for the Central Authority to be nonprofit making. However, if the variation in the damage costs is appropriately large, then no stable and efficient taxation schemes with zero benefit to the central Authority can exist. The paper concludes by examining the implications on the solution of the game when there is an imposition of constraints on the emission or treatment of effluents by the polluters.

\section{DESCRIPTION OF THE PROBLEM}

\section{The Parties}

We will, in general, make reference to the scheme of Figure 1 where the first block represents the pollution units called firms 


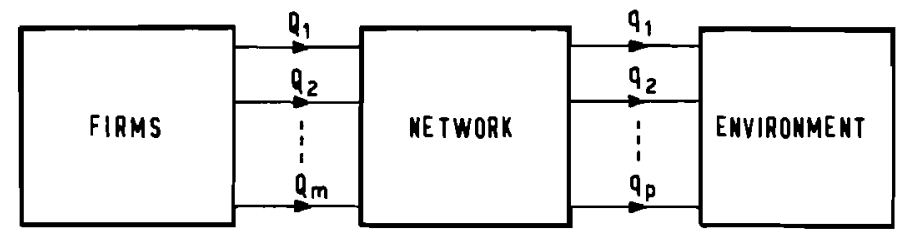

Figure 1. Structure of the system.

and the second and third blocks are the network of treatment plants and the environment (e.g. river basin, lake, sea,...). The variable $Q_{i}(i=1, \ldots, m)$ represents the mass flow rate of pollutant from firm $i$ to the network of treatment plants while the variable $q_{j}(j=1, \ldots, p)$ represents the mass flow rate of poliutant discharged by the jth effluent of the network into the environment.

Each firm $i$ is characterized by a profit $D_{i}\left(Q_{i}\right)$, i.e. the profit for producing an amount of good which corresponds to a production $Q_{i}$ of pollutant.

The network of treatment plants is characterized by a cost $T$ which is, in general, a function of the input and output vectors $Q=\left(Q_{1} \ldots Q_{m}\right)$ and $q=\left(q_{1} \ldots q_{p}\right)$, i.e.

$$
T=T(Q, q)
$$

Given a class of networks (i.e. given the structure of the network) we assume that the cost $T$ is the one corresponding to the least cost solution, i.e. Equation (1) represents the cost of the cheapest network in the class. For example, if the structure of the network is the one described in Figure 2a (completely disaggregated network: one firm - one plant - one effluent) then

$$
T=\sum_{i} T_{i}\left(Q_{i}, q_{i}\right)
$$

where $T_{i}\left(Q_{i}, q_{i}\right)$ is the cost of the cheapest treatment plant which transforms $Q_{i}$ into $q_{i}$. In this particular case the cost of the network is the sum of the single costs of the treatment plants. 

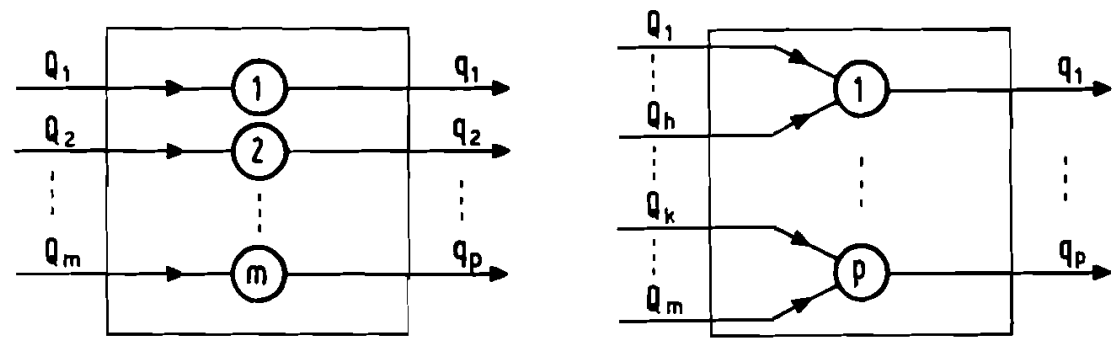

Figure 2. Two particular structures of the treatment network (each circle represents a wastewater treatment plant).

(a) completely disaggregated network

(b) partially disaggregated network.

On the other hand, if there is no restriction on the structure of the network, $T$ only depends upon the total input and the total output, i.e.

$$
T=T\left(\sum_{i} Q_{i}, \sum_{j} q_{j}\right)
$$

The third component of our system, the environment, is characterized by a function

$$
E=E(q)
$$

which is the sum, in monetary terms, of all possible damages to society (health, vegetation, goods,...).

In the following we will assume the existence of a unit called Central Authority (C.A.) which, depending upon the cases, will represent the treatment network and the environment or the environment alone (see Figure 3 ). In the first case (see Figure 3a) the C.A. is taking care of the construction of the network and will charge each firm $i$ depending upon the amount $Q_{i}$ of 


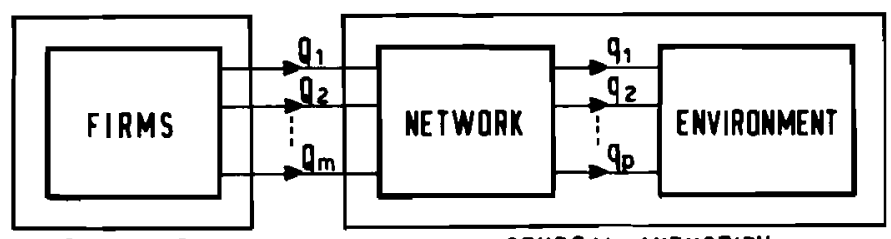

POLLUTERS

CENTRAL AUTHORITY

(a)

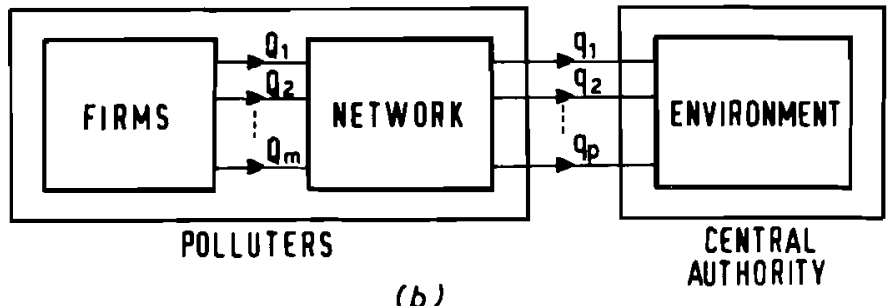

(b)

Figure 3. The unified representation

(a) charges on $Q_{i}$

(b) charges on $q_{j}$

pollutant produced, while in the second case (see Figure $3 \mathrm{~b}$ ) the charge is on the output $q_{j}$ of the network of treatment plants. These two cases can be formally described as a unique case (see Figure 4) where the units constituting the first component of the system are called polzuters and are supposed to

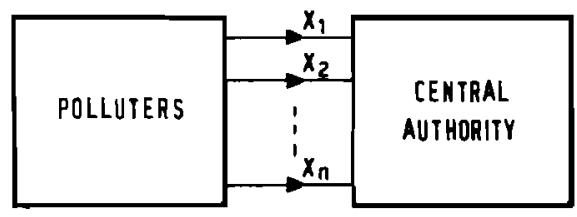

Figure 4. Compact structure of the system.

be characterized by a demand function $A_{i}\left(x_{i}\right)(i=1, \ldots, n)$ while the second component is the Central Authority which is characterized by a cost function $\mathrm{C}(\mathrm{x})$ where $\mathrm{x}$ stands for $\left(\mathrm{x}_{1}, \ldots, \mathrm{x}_{\mathrm{n}}\right)$. For example, if the situation is the one represented in Figure 
3a the polluters can be identified with the firms $\left(x_{i}=Q_{i}\right)$ and the demand and cost functions are

$$
\begin{aligned}
& A_{i}\left(x_{i}\right)=D_{i}\left(x_{i}\right) \\
& C(x)=\min _{q}[T(x, q)+E(q)] .
\end{aligned}
$$

On the other hand, if the charge is on the output of the treatment network (i.e. $x_{i}=q_{i}$ ) and the network is completely disaggregated as in Figure $2 a$, we have

$$
\begin{aligned}
& A_{i}\left(x_{i}\right)=\max _{Q_{i}}\left[D_{i}\left(Q_{i}\right)-T_{i}\left(Q_{i}, x_{i}\right)\right] \\
& C(x)=E(x)
\end{aligned}
$$

while when groups $y_{i}$ of firms jointly take care of their waste and discharge $q_{i}$, (i.e. the treatment network is only partially disaggregated as shown in Figure $2 \mathrm{~b}$ ), the demand functions are defined for each group $y_{i}$ as

$$
A_{i}\left(x_{i}\right)=\max _{\left\{Q_{j}\right\}}\left[\sum_{j \in y_{i}} D_{j}\left(Q_{j}\right)-T\left(\sum_{y_{i}} Q_{j}, x_{i}\right)\right] .
$$

In more complex situations the variable $x_{i}$ must be defined as a subvector of $\mathrm{q}$ and charges must be imposed on these subvectors. For the sake of simplicity in the following we will make reference only to the case in which $x_{i}$ is scalar, even if the theory presented in this paper can be developed for the general case.

From now on the set of polluters is called $N$, i.e.

$$
N=\{1,2, \ldots, n\}
$$


while by

$$
\bar{N}=\{0,1,2, \ldots, \mathrm{n}\}=N \cup\{0\}
$$

we denote the set of all parties (polluters and C.A.). Consistently, if $y$ is a subset of the parties, then $\bar{y}$ is the union of this subset and the C.A. i.e.

$$
\bar{y}=y \cup\{0\},
$$

while $y$ is the set obtained by eliminating the C.A. from $y$, i.e.

$$
\underline{y}=y-\{0\} .
$$

Notice that $\underline{y} \subseteq y \subseteq \bar{y}$ and either $\underline{y}=y$ or $\bar{y}=y$. Moreover, given a set $y$ of polluters we denote by $x^{y}$ the vector $\left\{x_{i}\right\}$ with $i \in y$ and for the sake of simplicity in notation we define the vector $\mathbf{x}^{y}$ also for sets $y$ containing the C.A. as $\mathbf{x}^{y}=\mathrm{x}^{y}$.

we can now define the aggregated demand function $A_{y}\left(x^{y}\right)$ for any set $y$ of polluters as

$$
A_{y}\left(x^{y}\right)=\sum_{i \in y} A_{i}\left(x_{i}\right)
$$

while for sets $y$ containing the $C$. A. we write

$$
\mathrm{A}_{y}\left(\mathrm{x}^{y}\right)=\mathrm{A}_{\underline{y}}\left(\mathbf{x}^{y}\right)
$$

since the C.A. is not characterized by any demand function. Similarly, given a set $y$ of polluters we define the cost $C_{y}\left(x^{y}\right)$ as the cost characterizing the C.A. in the case in which only the polluters of the set $y$ are present in the system, i.e.

$$
c_{y}\left(x^{y}\right)=c\left(x^{*}\right) \quad x^{*}: x^{* y}=x^{y}, x^{*-y}=0
$$


and again we write $C_{y}\left(x^{y}\right)$ instead of $C_{\underline{y}}\left(x^{\underline{y}}\right)$ in the case in which $y$ contains the $\mathrm{C}$. A.

The Characteristic Function

Given a system (i.e. a set $N$ of polluters, their demand functions, a Central Authority, and its cost function) we are interested in the maximum net profit attainable by any subset $y$ of the parties. This net profit, denoted by $\mathrm{V}(y)$ is the so-called characteristic function and is defined on all subsets $y$ of $\overline{i v}$ as follows

$$
\mathrm{V}(y)= \begin{cases}0 & \text { if } y=\underline{y} \text { or } y=\{0\} \\ \max _{\mathrm{x}^{y}}\left[A_{y}\left(\mathrm{x}^{y}\right)=\mathrm{C}_{y}\left(\mathrm{x}^{y}\right)\right] & \text { if } y=\bar{y} \neq\{0\}\end{cases}
$$

where $A_{y}\left(x^{y}\right)$ and $C_{y}\left(x^{y}\right)$ are defined as in Equations (2) and (3). In the following $x^{y}$ will denote that particular vector which solves the optimization problem (4). The assumption of zero net profit for all subsets of polluters $(y=\underline{y})$ represents the fact that polluters are not obliged to participate in the system but at the same time cannot enter the system without making an agreement with the C.A.

The reader accustomed to game theory must notice that we do not a priori assume that the characteristic function $V$ is superadditive $(V(\bar{x})+V(\bar{y}) \leq \mathrm{V}(\bar{x} \cup \bar{y})$ for all sets $\underline{x}$ and $\underline{y}$ of polluters such that $\underline{x} \cap \underline{y}=\emptyset$ ) as usually done in game theory (note the difference between $\mathrm{x}$ and $x$ ). Superadditivity can be a priori in ferred only if the option always exists for groups to act separately if their joint action would not lead to an improvement of their total profit. However in environmental pollution problems this option never exists, since the environmental damage can never be decomposed into the sum of individual damages each attributable to a given group.

An important attribute of characteristic functions is that of convexity. 
Definition 1 (Convexity of $\mathrm{V}$ )

A characteristic function is convex if

$$
\mathrm{V}(x \cup\{i\})-\mathrm{V}(x) \leq \mathrm{V}(y \cup\{i\})-\mathrm{V}(y)
$$

for all $x \subseteq y \subseteq \bar{N}$ and for all i $\in \bar{N}-y$.

\section{The Taxation Scheme}

The charge on $x_{i}$ is denoted by $C_{i}\left(x_{i}\right)$ and will often be referred to as tax in what follows. When the ith polluter is charged an amount $c_{i}\left(x_{i}\right)$ his benefit is given by

$$
B_{i}\left(x_{i}\right)=A_{i}\left(x_{i}\right)-C_{i}\left(x_{i}\right) \quad i \in N
$$

while the benefit accruing to the C.A. is

$$
\mathrm{B}_{0}(\mathrm{x})=\sum_{N} C_{i}\left(x_{i}\right)-C(x)
$$

We assume that each polluter is profit maximizing in the sense that he selects his discharge $x_{i}^{*}$ by maximizing Equation (6) i.e.

$$
\mathrm{B}_{i}^{*}=\max _{\mathrm{x}_{i}}\left[\mathrm{~B}_{i}\left(\mathrm{x}_{i}\right)\right]=\mathrm{B}_{i}\left(\mathrm{x}_{i}^{*}\right)
$$

Then the corresponding benefit for the C.A. is given by

$$
\mathrm{B}_{0}^{*}=\mathrm{B}_{0}\left(\mathrm{x}^{*}\right)
$$

We are now in the position to formally define a taxation scheme. Definition 2 (Taxation scheme)

A taxation scheme is a set of rules that, given a system, generates a set of taxes $C_{i}(\cdot), i \in N$.

Some examples of taxation schemes may clarify this definition. 


\section{EXAMPLE 1}

The following two rules define a taxation scheme.

1. Compute $V(\bar{N})$ and $x_{i} \bar{N}$.

2. Determine $\mathcal{C}_{i}(\cdot) \quad i \in N$ so that

$$
\begin{aligned}
& \text { (i) } \mathrm{x}_{\mathrm{i}}^{*}=\mathrm{x}_{\mathrm{i}}^{N} \quad \mathrm{i} \in \mathrm{N} \\
& \text { (ii) } \mathrm{B}_{\mathrm{i}}^{*}=\frac{1-\alpha}{\mathrm{n}} \mathrm{v}(\bar{N}) \quad 0 \leq \alpha \leq 1 \quad i \in N \text {. }
\end{aligned}
$$

Condition (i) means that the taxes are such that the polluters by solving their own problems will maximize the total (social) benefit since (i) implies

$$
\sum_{\bar{N}} B_{i}^{*}=V(N)
$$

Condition (ii) means that the polluters divide equally part of the total benefit. Moreover, if $\alpha=0$ there is no benefit for the C.A., while if $\alpha=1$ there is no benefit for the polluters.

$\underline{\text { Remark } 1}$

Rule 2 above makes sense only if the functions $C_{i}(\cdot)$ satisfying conditions ( $i$ ) and (ii) can actually be found. Obviously if the demand functions $A_{i}(\cdot)$ are concave then such functions $C_{i}(\cdot)$ exist and are characterized by

$$
\left.\frac{d C_{i}}{d x_{i}}\right|_{x_{i}^{\bar{N}}}=\left.\frac{\mathrm{dA}_{i}}{d x_{i}}\right|_{x_{i}^{\bar{N}}} \quad C_{i}\left(x_{i}^{\bar{N}}\right)=A_{i}\left(x_{i}^{\bar{N}}\right)-B_{i}^{*} .
$$

The first condition means that the marginal charge equals the marginal demand and implies proposition $2(i)$. The second condition which follows from Equation (6), leads to proposition 2(ii). The functions $C_{i}(\cdot)$ may not be everywhere differentiable as in the case of bulk or two-part tariffs. However, for our purpose it is sufficient to assume that $C_{i}(\cdot)$ is locally differentiable at $x_{i}^{\bar{N}}$. 
EXAMPLE 2 (Lexicographic taxation scheme)

The following two rules define a taxation scheme.

1. Given an ordering $i \rightarrow \omega(i)$ in $\bar{N}$ compute

$$
\mathrm{v}\left(x_{\mathrm{k}}\right) \text { for } x_{\mathrm{k}}=\{\mathrm{i}: \mathrm{w}(\mathrm{i}) \leq \mathrm{k}\} \quad \mathrm{k}=0,1,2, \ldots, \mathrm{n}
$$

(note that the computation of the last term $v\left(x_{n}\right)$ gives the vector $\mathrm{x}^{\bar{N})}$.

2. Determine $c_{i}(\cdot) \quad i \in N$ so that

$$
\begin{aligned}
& \text { (i) } \mathrm{x}_{i}^{*}=\mathrm{x}_{i}^{\bar{N}} \quad \mathrm{i} \in \mathrm{N} \\
& \text { (ii) } \mathrm{B}_{i}^{*}=\mathrm{V}\left(x_{w(i)}\right)-\mathrm{V}\left(x_{w(i)-1}\right) \quad i \in \bar{N} \\
& \mathrm{~V}\left(x_{-1}\right)=0 .
\end{aligned}
$$

As in the preceding example we have $\sum_{\bar{N}} B_{i}^{*}=V(\bar{N})$ (this follows from (i)). The sense of condition (ii) is as follows: a polluter gets a benefit equal to the improvement he generates in the total benefit when he enters the system following the order $\omega$. If $\omega(0)=0$ the C.A. has no benefit while if $\omega(0)=n$ we have the same scheme of Example 1 with $\alpha=1$.

\section{EXAMPLE 3 (Generalized taxation scheme).}

The following two rules define a taxation scheme.

1. As in rule 1 of Example 2, but for all orderings $\omega_{j}(\cdot)$, $j=1,2, \ldots,(n+1) !$

2. Determine $C_{i}(\cdot)$ i $\in N$ so that

$$
\text { (i) } \begin{aligned}
& x_{i}^{*}=x_{i}^{\bar{N}} \quad i \in N \\
& \text { (ii) } B_{i}^{*}=\sum_{j=1}^{(n+1) !} \lambda_{j}\left[v\left(x_{\omega_{j}(i)}\right)-v\left(x_{\omega_{j}}(i)-1\right)\right] \quad i \in \bar{N} \\
& 0 \leq \lambda_{j} \leq 1 \quad \sum_{j=1} \lambda_{j}=1 \quad v\left(x_{-1}\right)=0 .
\end{aligned}
$$


Again we have $\frac{\sum B_{i}^{*}}{N}=V(\bar{N})$. Condition (ii) says that the benefits of the ith polluter are a convex combination of the benefits obtained by means of all lexicographic taxation schemes.

EXAMPLE 4 (Shapley taxation scheme)

This scheme is a particular case of Example 3. In fact it corresponds to letting

$$
\lambda_{j}=\frac{1}{(n+1) !}
$$

in condition ( $i$ ).

Each example shows that a taxation scheme generates different taxes $C_{i}(\cdot)$ and different benefits $B_{i}^{*}$ when applied to different systems. For this reason $B_{i}^{\bar{y}}, i \in \bar{y}$ will indicate the benefit accruing to the ith party when the set of polluters in the system is $y$. Moreover, we define $B \frac{y}{i}=0, i \in \underline{y}$ since the polluters alone cannot have profit. Therefore, in the following, given a set $y \subseteq \bar{N}$, either $B_{i}^{y}=B_{i}^{\bar{y}}$ if $y=\bar{y}$, or $B_{i}^{y}=B_{i}^{\underline{y}}=0$ if $y=\underline{y}$.

\section{Attributes of Taxation Schemes}

As mentioned in the introduction three fundamental attributes can characterize taxation schemes, namely acceptability, efficiency and stability.

The acceptability of a taxation scheme corresponds to the fact that the benefits of all parties are non-negative. More precisely, we have the following definition.

Definition 3 (Acceptability)

A taxation scheme is acceptable, for the set $N$, if the corresponding vector of benefits $\mathrm{B}^{\bar{N}}=\left(\mathrm{B}_{0}^{\bar{N}}, 3_{1}^{\bar{N}}, \ldots, \mathrm{B}^{\mathrm{N}}\right)$ is non-negative, i.e.

$$
B_{i}^{\bar{N}} \geq 0 \quad \forall i \in \bar{N} .
$$


The notion of efficiency is directly related to the definition of characteristic function. In short, a taxation scheme is said to be efficient when the solution it generates (through the profit maximization of the polluters) is characterized by the maximum total benefit.

Definition 4 (Efficiency)

A taxation scheme is efficient with respect to $\bar{N}$ if

$$
\sum_{y} \mathrm{~B}_{i}^{y}=\mathrm{V}(y) \quad \forall y \subseteq \bar{N} .
$$

All the preceding examples are examples of acceptable and efficient taxation schemes.

The literature on "optimal taxing" (see, for instance, [1-3]) has extensively dealt with the problem of selecting charges such that the total cost of treatment is minimized while a given water quality standard is satisfied. If we define the environmental cost $\mathrm{E}(\mathrm{q})$ as zero when the standard is met and infinity when it is not, we see that the classical problem is reduced to the determination of a particular efficient taxation scheme.

Finally, stability is defined as follows.

Definition 5 (stability)

A taxation scheme is stable, with respect to $\bar{N}$, if

$$
\sum_{y} B_{i}^{\bar{N}} \geq \sum_{y} B_{i}^{y} \quad \forall y \subseteq \bar{N} .
$$

That is, we have a stable taxation scheme when all subsets $y$ of $\bar{N}$ take advantage of the coalition with the remaining parties. If a taxation scheme is efficient with respect to $\bar{N}$, we have

$$
\sum_{y} \mathrm{~B}_{i}^{y}=\mathrm{V}(y) \quad \forall y \subseteq \bar{N}
$$


Therefore, in this case the condition of stability can be modified as follows.

Remark 2

An efficient taxation scheme is stable with respect to $\bar{N}$, if

$$
\sum_{y} \mathrm{~B}_{\mathrm{i}}^{\bar{N}} \geq \mathrm{V}(y) \quad \forall y \subseteq \bar{N} .
$$

We now use two of the preceding notions (efficiency and stability) to give the following definition.

Definition 6 (Core)

The set of the vectors $B^{\bar{N}}$ of benefits generated by all the taxation schemes which are efficient and stable with respect to $\bar{N}$ is called the core of $\bar{N}$.

A simple and visual representation of the core can be given in the case $\mathrm{n}=2$, i.e. when there are only two polluters in the system. In Figure 5 the three dimensional space of benefits $\left(B_{0}^{*}, B_{1}^{*}, B_{2}^{*}\right)$ is shown, together with the three planes

$$
\begin{aligned}
& \mathrm{B}_{0}^{*}+\mathrm{B}_{1}^{*}=\mathrm{V}(\{0,1\}) \\
& \mathrm{B}_{0}^{*}+\mathrm{B}_{2}^{*}=\mathrm{V}(\{0,2\}) \\
& \mathrm{B}_{0}^{*}+\mathrm{B}_{1}^{*}+\mathrm{B}_{2}^{*}=\mathrm{V}(\{0,1,2\})
\end{aligned}
$$

These planes are characterized by the fact that they contain all the vectors of benefits generated by efficient taxation schemes. More precisely, if an efficient taxation scheme is applied to $\bar{N}$ the corresponding vector of benefits belongs to the last plane, while, if it is applied to, for example, $\{0,1\}$ the corresponding vector of benefits lies on the intersection of the first plane with the plane $\mathrm{B}_{2}^{*}=0$. 


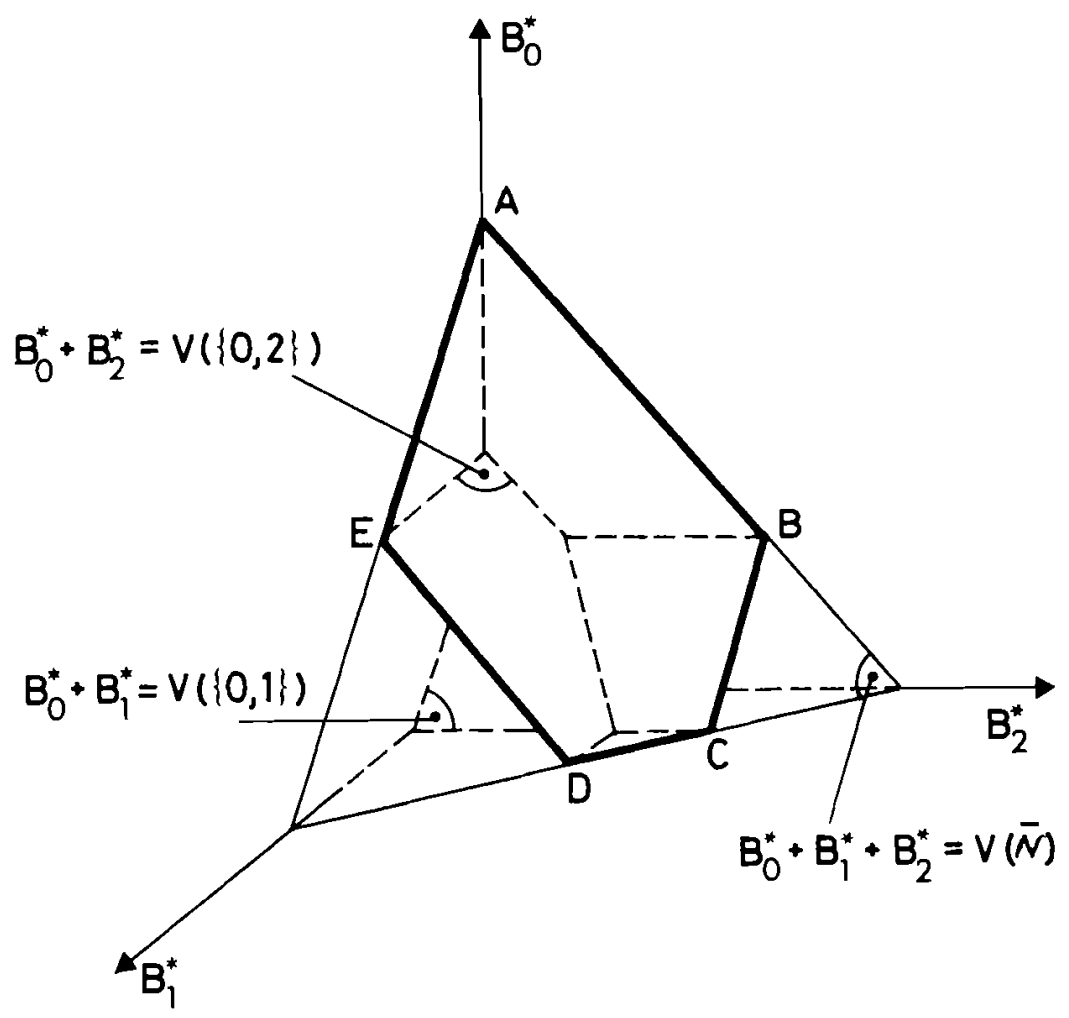

Figure 5. The space of the benefits and the core (ABCDE).

It can be noticed from the figure that $V(\{0,1,2\})$ is greater than $V(\{0,1\})$ and $v(\{0,2\})$, which means that in this case the characteristic function is convex (see Equation (5)). Moreover, the vectors $B^{*}$ corresponding to the points of the polyhedron $A B C D E$, are such that

$$
\sum_{\bar{N}} \mathrm{~B}_{\mathrm{i}}^{*}=\mathrm{V}(\overline{\mathrm{N}}), \quad \sum_{y} \mathrm{~B}_{\mathrm{i}}^{*} \geq \mathrm{V}(y) \quad y \subseteq \bar{N}
$$


and there are no other points satisfying these relationships. Since these are the conditions of efficiency and stability it means that the core is contained in the convex polyhedron ABCDE. In the following we will prove that there exist taxation schemes generating all the points of the polyhedron $A B C D E$ (see Property 3 below) so that we will conclude that the core is the convex polyhedron $A B C D E$. The following three properties will be used later on to analyze some particular cases. The first two are very simple while the third is a suitable reformulation of an important result proved by shapley [4].

\section{Property 1}

A taxation scheme which is stable and efficient with respect to

$\bar{N}$ is also acceptable with respect to $\bar{N}$.

Proof

Stability and efficiency imply (see Remark 2)

$$
\sum_{y} B_{i}^{\bar{N}} \geq V(y) \quad \forall y \subseteq \bar{N}
$$

On the other hand from the definition of the characteristic function we get

$$
V(\{i\})=0 \quad \forall i \in \bar{N}
$$

so that Equation (9) gives

$$
\mathrm{B}_{\mathrm{i}}^{\bar{N}} \geq 0 \quad \forall i \in \bar{N}
$$

which means acceptability of the taxation scheme.

$\square$

\section{Property 2}

If the demand functions $A(\cdot)$ are concave functions, then a necessary and sufficient condition for the existence of a stable and efficient taxation scheme is 


$$
\mathrm{v}(\bar{N}) \geq \mathrm{v}(y) \quad \forall y \subseteq \bar{N} .
$$

Proof

\section{Necessity}

The efficiency of the taxation scheme implies

$$
V(\bar{N})=\sum_{\bar{N}} B_{i}^{\bar{N}}=\sum_{y} B_{i}^{\bar{N}}+\sum_{\bar{N}-y} B_{i}^{\bar{N}} .
$$

Thus, from Property 1 we obtain

$$
\mathrm{V}(\bar{N}) \geq \sum_{y} \mathrm{~B}_{i}^{\bar{N}}
$$

which can be transferred into

$$
\mathrm{V}(\bar{N}) \geq \mathrm{V}(y)
$$

if Remark 2 is taken into account.

\section{Sufficiency}

The concavity of the functions implies (see Remark 1) the existence of an efficient taxation scheme of the kind described in Example 1. Let us select the taxation scheme with $\alpha=1$, i.e. the taxation scheme for which $B_{i}^{\bar{N}}=0$ for all polluters $i$ and ${ }_{B}^{N}=V(\bar{N})$. For this scheme we have

$$
\sum_{y} B_{i}^{\bar{N}}= \begin{cases}0 & \text { if } y=\underline{y} \\ V(\bar{N}) & \text { if } y=\bar{y} .\end{cases}
$$

But, by assumption, $\mathrm{V}(\bar{N}) \geq \mathrm{V}(y) \quad \forall y \subseteq \bar{N}$ (and therefore $\mathrm{V}(\bar{N}) \geq \mathrm{V}(y) \quad \forall y=\bar{y}$ while for $y=\underline{y}$ we have $\mathrm{V}(y)=0$ ) so that

$$
\sum_{y} \mathrm{~B}_{i}^{\bar{N}} \geq \mathrm{V}(y) \quad \forall y \subseteq \bar{N}
$$


which is the stability condition for efficient taxation schemes (see Remark 2).

Property 3

If the characteristic function is convex, the core exists and is a convex polyhedron. Moreover the generalized lexicographic taxation schemes (see Example 3) are stable (and efficient) and generate all the benefits of the core.

Proof

A fundamental theorem due to Shapley [4] says that, if the characteristic function $V(y)$ is convex the set $C$ of the $(n+1)$ th dimensional vectors $B=\left(B_{0}, B_{1}, \ldots, B_{n}\right)$ satisfying the following two conditions

$$
\sum_{\bar{N}} B_{i}=V(\bar{N})
$$

and

$$
\sum_{y} \mathrm{~B}_{i} \geq \mathrm{V}(y) \quad \forall y \subseteq \bar{N},
$$

is a convex polyhedron characterized by $(n+1)$ ! vertices. Moreover, let $w$ represent one out of the $(n+1)$ ! ordering $i \rightarrow \omega(i)$ of the components of the $(n+1)$ th dimensional vector $B$ and define $B^{\omega}$ as

$$
B^{\omega} \triangleq\left(B_{0}^{w} \cdots B_{n}^{\omega}\right)
$$

where

$$
\begin{aligned}
& \mathrm{B}_{\mathrm{i}}^{\omega} \triangleq \mathrm{V}\left(x_{\omega(\mathrm{i})}\right)-\mathrm{V}\left(x_{\omega(\mathrm{i})-1)}\right. \\
& x_{\mathrm{k}} \triangleq\{\mathrm{i}: \omega(\mathrm{i}) \leq \mathrm{k}\} \quad \mathrm{V}\left(x_{-1}\right)=0 .
\end{aligned}
$$


Then, the point $B^{\omega}$ is one of the vertices of the set $C$. If we compare the preceding equations with the taxation scheme defined in Example 2 we understand that the lexicographic taxation schemes are stable and efficient, and that the generalized schemes generate all benefits belonging to $c$, i.e. the core and the set $C$ coincide.

PROPERTIES OF THE MODEL

In order to analyze the existence of stable and efficient taxation schemes in regional environmental management, it is necessary to postulate some structural properties for the functions characterizing the units we called firms, treatment network and environment.

\section{Profit Functions}

We assume that the profit function $D_{i}(\cdot)$ has the following properties:

$$
\begin{array}{ll}
\text { (i) } \mathrm{D}_{i}(0)=0 & \\
\text { (ii) } \mathrm{dD}_{i} / \mathrm{dQ} Q_{i}>0 & Q_{i}>0 \\
\text { (iii) } \mathrm{d}^{2} \mathrm{D}_{i} / d Q_{i}^{2}<0 & Q_{i}>0 .
\end{array}
$$

Assumptions (i) and (ii) only state that no production implies no profit and that more production implies more profit. Assumption (iii), namely the fact that the marginal profit is a decreasing function of $Q_{i}$, is usually satisfied for sufficiently high values of $Q_{i}$, i.e., for sufficiently large firms. On the other hand, small firms are sometimes characterized by increasing marginal profits because of the economies of scale in the technology of production. This means that the theory developed in the following can only be applied to the cases when the firms 
exploiting the common resource are so large that their marginal profit cannot be increased by increasing the amount of goods produced. On the other hand point (iii), namely that the profit function $D_{i}(\cdot)$ is concave, cannot be relaxed since it implies that efficient taxation schemes can be levied on $Q_{i}$ (see Remark 1).

\section{Treatment Plant Cost}

The cost function $T_{i}\left(Q_{i}, q_{i}\right)$ of a single treatment plant is assumed to exhibit the following properties $([1,3,5])$ :

$$
\begin{aligned}
& \text { (a) } T_{i}\left(Q_{i}, q_{i}\right) \text { is convex with respect to } q_{i} \\
& \text { (b) } T_{i}\left(Q_{i}, q_{i}\right) \text { is convex with respect to } Q_{i} \\
& \text { (c) } T_{i}\left(Q_{i}, a Q_{i}\right) \text { is concave with respect to } Q_{i}
\end{aligned}
$$

These properties are always satisfied particularly the third one which is due to the economies of scale always present to some extent. Thus the function $T_{i}\left(Q_{i}, q_{i}\right)$ is not convex (see property (c)) though it is convex with respect to $Q_{i}$ and $q_{i}$. The shape of the surface $T_{i}\left(Q_{i}, q_{i}\right)$ is in fact of the $k$ ind shown in Figure 6.

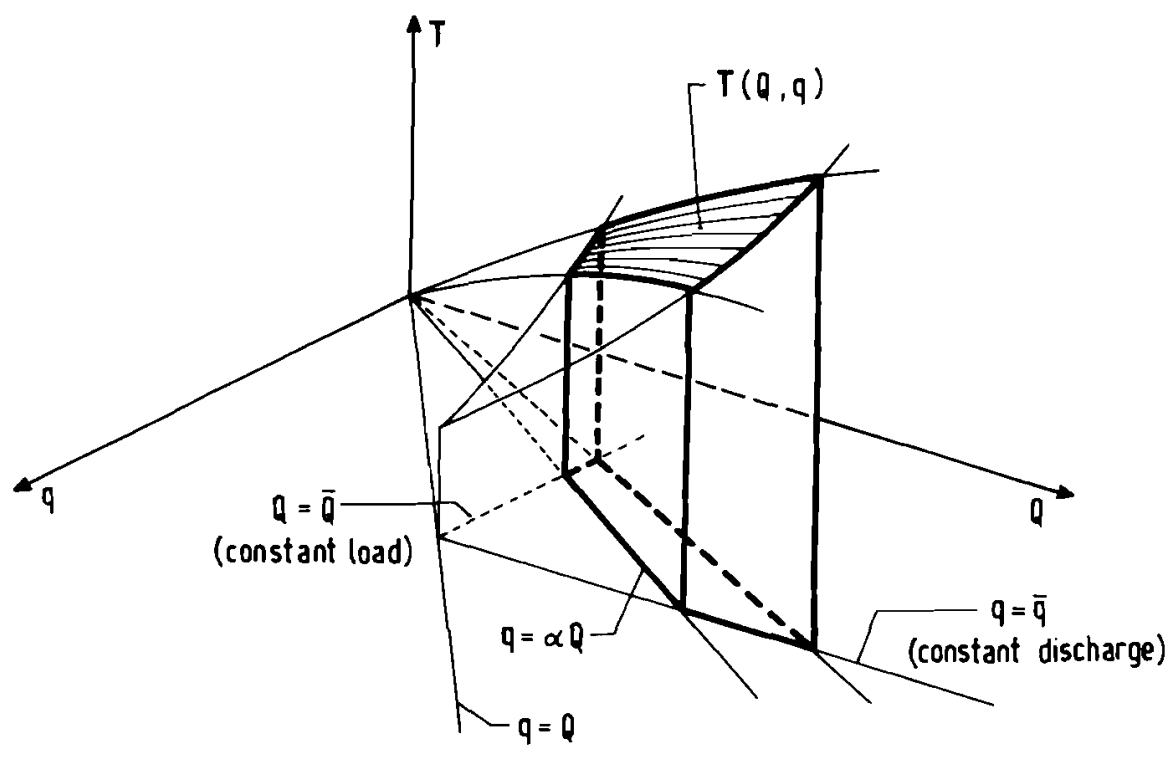

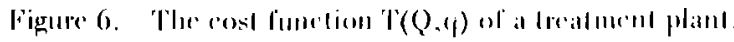




\section{Treatment Network Cost}

Let us assume that there are no constraints on the structure of the network. Thus, as we already said, the cost of the treatment network is a function $T\left(Q_{+}, q_{+}\right)$of the total input $Q_{+}=\sum_{i} Q_{i}$ of the network and of its total output $q_{+}=\sum_{j} q_{j}$. The cost of a treatment network will be assumed to satisfy an important property referred to as economies of scale which can be formulated as follows :

$$
T\left(Q_{+}^{\prime}+\Delta, q_{+}^{\prime}+\delta\right)-T\left(Q_{+}^{\prime}, q_{+}^{\prime}\right) \geq T\left(Q_{+}^{\prime \prime}+\Delta, q_{+}^{\prime \prime}+\delta\right)=T\left(Q_{+}^{\prime \prime}, q_{+}^{\prime \prime}\right)
$$

for

$$
Q_{+}^{\prime}<Q_{+}^{\prime \prime} \quad q_{+}^{\prime}<q_{+}^{\prime \prime} \quad \delta<\Delta .
$$

This property is a natural generalization of the property of economies of scale of the cost of a treatment plant (see property (c) above). In fact if it is assumed that

$$
q_{+}^{\prime}=\alpha Q_{+}^{\prime} \quad q_{+}^{\prime \prime}=\alpha Q_{+}^{\prime \prime} \quad \delta=\alpha \Delta
$$

Equation (10) becomes

$$
\mathrm{T}\left(Q_{+}^{\prime}+\Delta, \alpha\left(Q_{+}^{\prime}+\Delta\right)\right)-\mathrm{T}\left(Q_{+}^{\prime}, \alpha Q_{+}^{\prime}\right) \geq \mathrm{T}\left(Q_{+}^{\prime \prime}+\Delta, \alpha\left(Q_{+}^{\prime \prime}+\Delta\right)\right)-\mathrm{T}\left(Q_{+}^{\prime}, \alpha Q_{+}^{\prime \prime}\right)
$$

from which it follows that the function $T\left(Q_{+}, \alpha Q_{+}\right)$is concave. Moreover, if the structure of the network of the treatment plant is somehow constrained, the property of economies of scale must be formulated in a more general way, by substituting $Q_{+}^{\prime}, q_{+}^{\prime}, Q_{+}^{\prime \prime}$, $q_{+}^{\prime \prime}, \Delta$ and $\delta$ in Equation (10) with the vectors $Q^{\prime}, q^{\prime}, Q^{\prime \prime}, q^{\prime \prime}, \Delta$ and $\delta(\Delta \geq 0, \delta \geq 0)$. 


\section{Environmental Damage}

The main feature to be taken into account when discussing the damages produced by the users of an environmental resource is the so called congestion effect. At some low level of use an additional use of the resource may practically generate no surplus of damage. A point is reached, however, where an additional user will cause others to have to incur additional costs or suffer disutilities associated with congestion (see [6] for details). This property can be given the following very general form

$$
E\left(q^{\prime}+\delta\right)-E\left(q^{\prime}\right) \leq E\left(q^{\prime \prime}+\delta\right)-E\left(q^{\prime \prime}\right)
$$

where $q^{\prime}, q^{\prime \prime}$ and $\delta$ are three non-negative vectors and $q^{\prime \prime} \geq q^{\prime}$.

Equation (11) implies that $\mathrm{E}$ is convex with respect to each component $q_{i}\left(q_{j}^{\prime}=q_{j}^{\prime \prime}\right.$ and $\delta_{j}=0$ for all $j \neq i$ in Equation (11)).

\section{ANALYSIS OF PARTICULAR CASES}

The traditional approach to pollution control has been legislative regulation of the discharges. The laws existing today either stipulate the allowable amount of waste that each type of firm can discharge (effluent standard) or fix a required treatment efficiency (e.g. $85 \%$ biological oxygen demand removal before discharge). In some environmental laws it is tacitly assumed that all the discharges satisfying the standards induce negligible environmental damage, while other legislations do not consider $\mathrm{E}(\mathrm{q})$ to be zero and therefore each polluter is asked to compensate the damage in monetary terms. Generally, each polluter acts by himself and takes care of his own treatment plant. However, a regional waste treatment system could often be of advantage because of the economies of scale and the possibility of reallocating discharges. Thus, it is of interest to analyze the case of a regional authority (the C.A.) which takes care of the treatment network. In doing so the C.A. either will acquire the rights of discharge owned by the firms (in the case of effluent 
standard) or will be obliged to use a treatment network of sufficiently high efficiency (in the case of required treatment efficiency). In both cases taxes are levied on the pollutant flow rates $Q_{i}$ generated by each firm.

In this section we analyze the existence of stable and efficient taxation schemes, both in the case of effluent standards and in the case of fixed treatment efficiency. To gain insight into the advantages of a well-designed regional waste treatment network we will make a comparison between the case in which the treatment network is unconstrained and the case in which it must be a completely disaggregated one.

A more recent approach to water quality control is by effluent charges. The stability analysis of this alternative possibility will be developed later.

\section{Effluent Standards and Taxes on $Q_{i}$}

We assume that each firm has a right of discharge $\bar{q}_{i}$ and that the Central Authority is taking care of the treatment facilities. When no constraints are imposed on the structure of the network the total cost of the C.A. is

$$
\begin{aligned}
& C(Q)=\min _{q}\left[T\left(Q_{+}, q_{+}\right)+E(q)\right] \\
& q_{+} \leq \bar{q}_{+}^{\bar{N}}=\sum_{N} \bar{q}_{i} .
\end{aligned}
$$

The last constraint means that the C.A. can discharge up to a maximum $\overline{\mathrm{q}}_{+}^{\bar{N}}$ given by the sum of the rights of discharge of the polluters. In general, the optimal solution $q^{\circ}$ is a function of $Q_{+}$, i.e.

$$
q^{\circ}=q^{\circ}\left(Q_{+}\right)
$$

while if the environmental damage $\mathrm{E}(\mathrm{q})$ is assumed to be zero 
the total output $q_{+}^{\circ}$ is not dependent upon $Q$, since it is obviously given by

$$
\mathrm{q}_{+}^{\circ}=\overline{\mathrm{q}}_{+}^{\bar{N}}
$$

When the central Authority is constrained to use a completely disaggregated network we assume that it also loses the right to reallocate the discharges of the polluters, i.e. the total cost of the C.A. is

$$
C(Q)=\min _{q}\left[\sum_{N} T_{i}\left(Q_{i}, q_{i}\right)-E(q)\right]
$$

subject to

$$
\mathrm{q}_{i} \leq \overline{\mathrm{q}}_{\mathrm{i}} \quad \forall i \in N \quad
$$

In this case the vector $q^{\circ}$ is a function of the vector $Q$ (and not of $Q_{+}$), while if $\mathrm{E}(\mathrm{q})=0$ obviously $\mathrm{q}_{i}^{\circ}=\overline{\mathrm{q}}_{i}$.

We analyze now four possible cases in the following order:

(i) unconstrained network, $\mathrm{E}(\mathrm{q})=0$

(ii) unconstrained network, $E(q) \neq 0$

(iii) completely disaggregated network, $E(q)=0$

(iv) completely disaggregated network, $E(q) \neq 0$.

(i) Under the hypothesis $\mathrm{E}(\mathrm{q})=0$, we can prove the following basic property.

\section{Property 4}

If the environmental damage is negligible and the cost of the treatment network satisfies Equation $(10)$, then there exist efficient and stable taxation schemes.

The proof of this property, given in the Appendix, can be easily modified in order to show that if the strict inequality is satisfied in Equation (10), then there exists an infinity of stable 
taxation schemes characterized by $B_{0}=0$ (see segment $\mathrm{CD}$ of Figure 5). In other words, the central Authority can charge the firms only for the cost of treating the waste and still have options in sharing the total benefit among the firms.

(ii) If we assume

$$
\mathrm{E}(\mathrm{q}) \neq 0 \quad \mathrm{q}>0
$$

it is not possible to prove that the characteristic function is convex and therefore the existence of stable taxation schemes cannot be inferred any more by means of Property 3 . On the other hand, it could be shown by means of simple examples that the necessary and sufficient condition of Property 2 can be either satisfied or not, so that we can have cases in which stable taxation schemes exist and cases in which all taxation schemes are unstable. But, even when stable taxation schemes exist, the C.A. is more constrained than in the preceding case $(E(q)=0)$. In fact we will now prove that when the congestion effect of the environment is particularly important taxation schemes characterized by zero profit of the C.A. cannot be stable. This means that stability can be obtained only at the price of transforming the C.A. into a profit corporation. More precisely, we prove the following property.

\section{Property 5}

When the congestion effect is dominant with respect to the economies of scale (in the sense specified by Equation (12) below), each polluter desires to expel the others from the system, unless the C.A. is to some extent a profit-making corporation.

Let us denote with $q^{\bar{N}}$ the output vector of the treatment network serving the system $N\left(q^{\bar{N}}=q^{\circ}\left(Q_{+}^{\bar{N}}\right)\right)$ and with $q^{\{i\}}$ the output vector of the treatment network that would be used if the ith polluter were alone in the regional system with a pollutant production $Q_{i}^{\bar{N}}\left(q^{\{i\}}=q^{\circ}\left(Q_{i}^{N}\right)\right)$. Then the reduction of the treatment cost due to the economies of scale is 


$$
\sum_{N} \mathrm{~T}\left(Q_{i}^{\bar{N}}, \mathrm{q}_{+}^{\{i\}}\right)-\mathrm{T}\left(Q_{+}^{\bar{N}}, \mathrm{q}_{+}^{\bar{N}}\right)
$$

while

$$
E\left(q^{\bar{N}}\right)-\sum_{N} E\left(q^{\{i\}}\right)
$$

is the increase of the environmental damage due to the congestion effect. The congestion effect is dominant with respect to the economies of scale if

$$
\mathrm{E}\left(\mathrm{q}^{\bar{N}}\right)-\sum_{N} \mathrm{E}\left(\mathrm{q}^{\{i\}}\right)>\sum_{N} \mathrm{~T}\left(Q_{i}^{\bar{N}}, \mathrm{q}_{+}^{\{i\}}\right)-\mathrm{T}\left(\mathrm{Q}_{+}^{\bar{N}}, \mathrm{q}_{+}^{\bar{N}}\right)
$$

Thus Property 5 is equivalent to the following precise statements: Equation (12) implies the non-existence of stable and efficient taxation schemes with ${ }_{0}^{\bar{N}}=0$.

\section{Proof of Property 5}

The characteristic function is given by

$$
\mathrm{V}(\bar{N})=\mathrm{A}_{\bar{N}}\left(\mathrm{Q}^{\bar{N}}\right)-\left[\mathrm{T}\left(\mathrm{Q}_{+}^{\bar{N}}, \mathrm{q}_{+}^{\bar{N}}\right)+\mathrm{E}\left(\mathrm{q}^{\bar{N}}\right)\right]
$$

Hence, from Equation (12) we obtain

$$
V(\bar{N})<\sum_{N}\left[A_{i}\left(Q_{i}{ }^{\bar{N}}\right)-T\left(Q_{i}^{\bar{N}} \cdot q_{+}{ }^{\{i\}}\right)-E_{i}\left(q^{\{i\}}\right)\right] \leq \sum_{N} V_{i}
$$

where

$$
\mathrm{v}_{\mathrm{i}}=\mathrm{V}(\{0, \mathrm{i}\})
$$

On the other hand if there exists a taxation scheme which is stable and efficient with respect to $\bar{N}$ we have

$$
\mathrm{B}_{0}^{\bar{N}}+\mathrm{B}_{\mathrm{i}}^{\bar{N}} \geq \mathrm{v}_{\mathrm{i}} \quad \forall \mathrm{i} \in N .
$$


Thus,

$$
\sum_{N}\left[B_{0}^{\bar{N}}+B_{i}^{\bar{N}}\right] \geq \sum_{N} v_{i}
$$

from which the following sequence of expressions can be obtained:

$$
\begin{aligned}
& \mathrm{nB}_{0}^{\bar{N}}+\sum \mathrm{B}_{i}^{\bar{N}} \geq \sum_{N} \mathrm{v}_{i} \\
& (\mathrm{n}-1) \mathrm{B}_{0}^{\bar{N}}+\mathrm{V}(\bar{N}) \geq \sum_{N} \mathrm{v}_{i} \\
& \mathrm{~B}_{0}^{\bar{N}} \geq\left[\sum_{N} \mathrm{v}_{i}-\mathrm{V}(\bar{N})\right] /(\mathrm{n}-1) .
\end{aligned}
$$

But Equation (13) implies that the right hand side of the last expression is strictly positive so that the property is proved. $\square$ From the proof of this property we can conclude that if we consider a sequence of problems in which the environment is more and more sensitive to the congestion effect we would obtain cores that are smaller and smaller. In Figure 7 a sequence (a) - (d) is shown; in (a) and (b) inequality (12) is not satisfied, while (c) refers to the limit case in which Equation (12) is satisfied with the equality sign.

(iii) Let us now analyze the implications of constraining the C.A. to use a completely disaggregated network of treatment plants. For this, consider first the case in which the environmental damage is negligible

$$
E(q)=0
$$

and recall that in this case $q_{i}^{\circ}=\bar{q}_{i}$. Then

$$
V(\bar{N})=\max _{Q}\left[\sum_{N} A_{i}\left(Q_{i}\right)-\sum_{N} T_{i}\left(Q_{i}, \bar{q}_{i}\right)\right]=\sum_{N} \max _{Q_{i}}\left[A_{i}\left(Q_{i}\right)-T_{i}\left(Q_{i}, \bar{q}_{i}\right)\right]
$$


i.e.

$$
\mathrm{V}(\bar{N})=\sum_{N} \mathrm{~V}_{i}
$$

and this condition implies that the characteristic function is convex. In fact, since $v_{i}$ is strictly positive, from Equation (15) we obtain

$$
\mathrm{V}(x) \leq \mathrm{V}(y) \quad \forall x \subseteq y
$$

which implies that condition (5) is satisfied with the strict inequality if $i=0$ and with the equal sign if $i \neq 0$. Since $v$
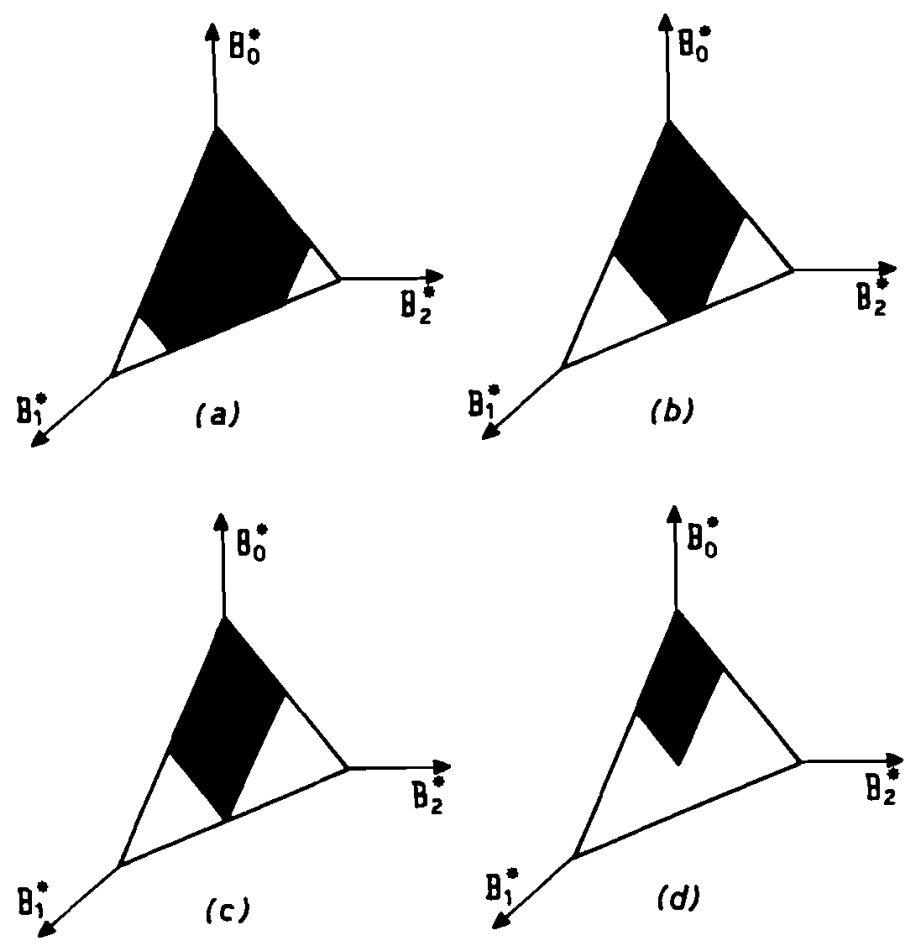

Figure 7. Smaller cores are obtained for increasing congestion effect. 
is convex, Property 3 can be applied and the conclusion is that the core exists. The fundamental difference with respect to the case of the aggregated network is that we now have only one point in the core with $B_{0}^{\bar{N}}=0$. In fact if $B_{0}^{\bar{N}}=0$ we have

$$
V(\bar{N})=\sum_{\bar{N}} B_{i}^{\bar{N}}=\sum_{N} B_{i}^{\bar{N}} .
$$

Hence stability and Equation (15) imply

$$
B_{i}^{\bar{N}}=v_{i}
$$

which proves the uniqueness of such taxation schemes. This case is shown in Figure 8 where the point $C=D$ represents the unique

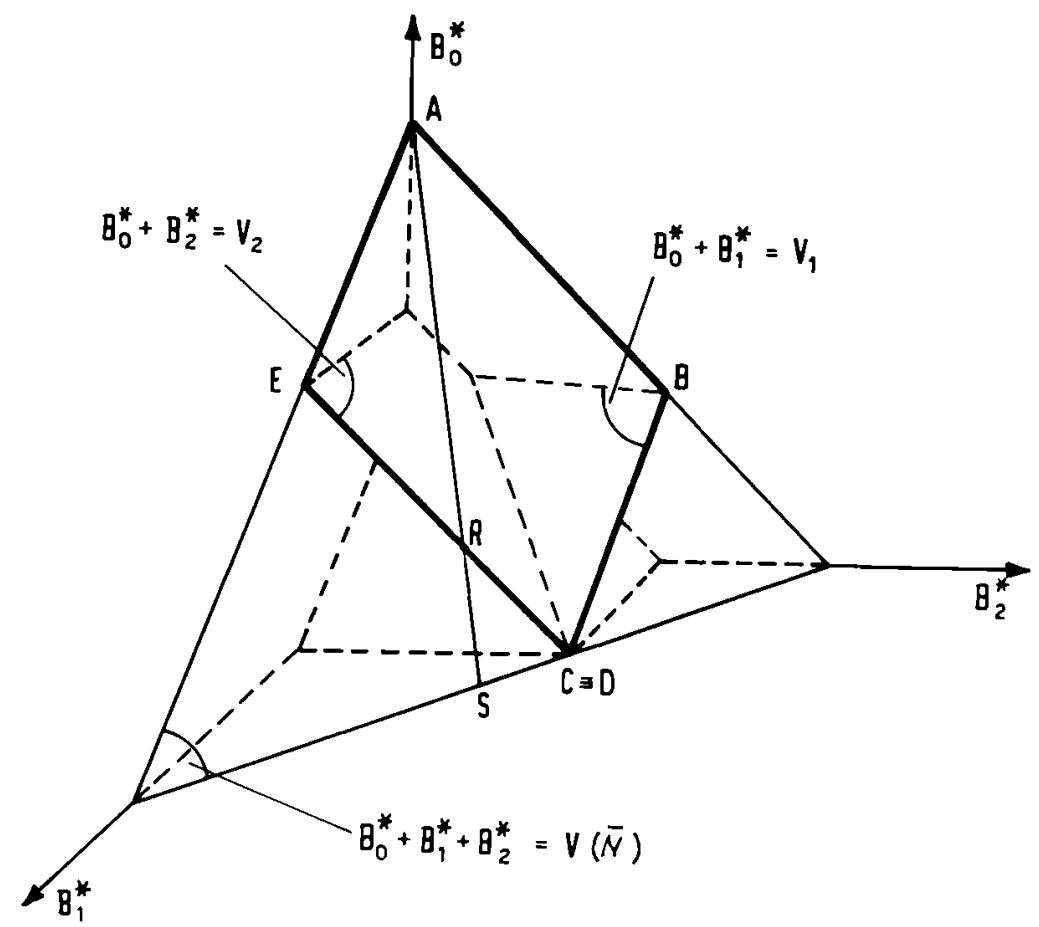

Figure 8 . The core when $\mathrm{E}(\mathrm{q})=0$ and a completely disaggregated network is used. 
possibility for the C.A. to be a non-profit corporation. This point requires a specific distribution of the benefit $V(\bar{N})$ among the firms. If a different distribution is desired (see, for instance, point $S$ of Figure 8 ), this can be done only by means of an unstable taxation scheme. Nevertheless, if the benefit of the C.A. is allowed to be positive then the scope can be obtained by means of stable taxation schemes (see segment AR of Figure 8 ).

(iv) We finally consider the case

$$
\mathrm{E}(\mathrm{q}) \neq 0 \quad \mathrm{q}>0
$$

with the C.A. constrained to use a completely disaggregated network of treatment plants. Again as in the case of aggregated networks we can have cases in which there are no stable taxation schemes. This can happen when there exists a subset $x$ of $\bar{N}$ such that the variation of the damage produced by the set $\bar{N}-x$ is greater than the variation of the net benefit

$$
w(\bar{N})=\sum_{N}\left[A_{i}\left(Q_{i}^{\bar{N}}\right)-T_{i}\left(Q_{i}^{\bar{N}}, q_{i}^{\bar{N}}\right)\right]
$$

i.e. when

$$
E\left(q^{\bar{N}}\right)-E\left(q^{\bar{x}}\right)>w(\bar{N})-w(\bar{x})
$$

where

$$
q^{\bar{N}}=q^{\circ}\left(Q^{\bar{N}}\right) \quad q^{\bar{x}}=q^{\circ}\left(Q^{\bar{x}}\right) .
$$

In fact condition (16) implies

$$
\mathrm{V}(\bar{N})=\mathrm{W}(\bar{N})-\mathrm{E}\left(\mathrm{q}^{\bar{N}}\right)<\mathrm{W}(\bar{x})-\mathrm{E}\left(\mathrm{q}^{\bar{x}}\right)=\mathrm{V}(\bar{x})
$$

i.e.

$$
\mathrm{V}(\bar{N})<\mathrm{V}(\bar{x})
$$


which contradicts the necessary condition (see Property 2) for the existence of stable and efficient taxation schemes. On the other hand if the sign $\geq$ holds in Equation (16) for all $x \in N$ we can prove that

$$
V(\vec{N}) \geq V(\bar{x})
$$

which implies the existence of the core. The only difference with respect to the case of unconstrained networks (see point (ii) above) is the uniqueness of the stable and efficient taxation scheme which assigns a zero benefit to the C.A., while the difference with respect to the preceding case (iii) is the possibility of non-existence of such particular taxation schemes. This easily follows from Equation (14) and the condition

$$
\mathrm{V}(\bar{N}) \leq \sum_{\hat{N}} \mathrm{~V}_{i}
$$

which can be proved with the use of the property of congestion effect of the environment. In Figure 8 an example is shown with $\mathrm{V}(\bar{N})=\sum_{N} \mathrm{~V}_{i}$, while in Figure 9 (notice that $\mathrm{V}(\bar{N})<\sum_{N} \mathrm{~V}_{i}$ ) the lowest point of the core (point $C \equiv D$ ) is characterized by

$$
B_{0}^{\bar{N}}=\left[\sum_{N} V_{i}-V(\bar{N})\right] /(n-1)
$$

which corresponds to the limit given by Equation (14).

In order to complete our analysis, we could now consider the case in which the Central Authority can reallocate the rights of discharge even when it is forced to use a completely disaggregated network. The cost of the C.A. is then

$$
\begin{aligned}
& C(Q)=\min _{q}\left[\sum_{N} T_{i}\left(Q_{i}, q_{i}\right)-E(q)\right] \\
& q_{+} \leq \bar{q}_{+}^{\bar{N}} .
\end{aligned}
$$




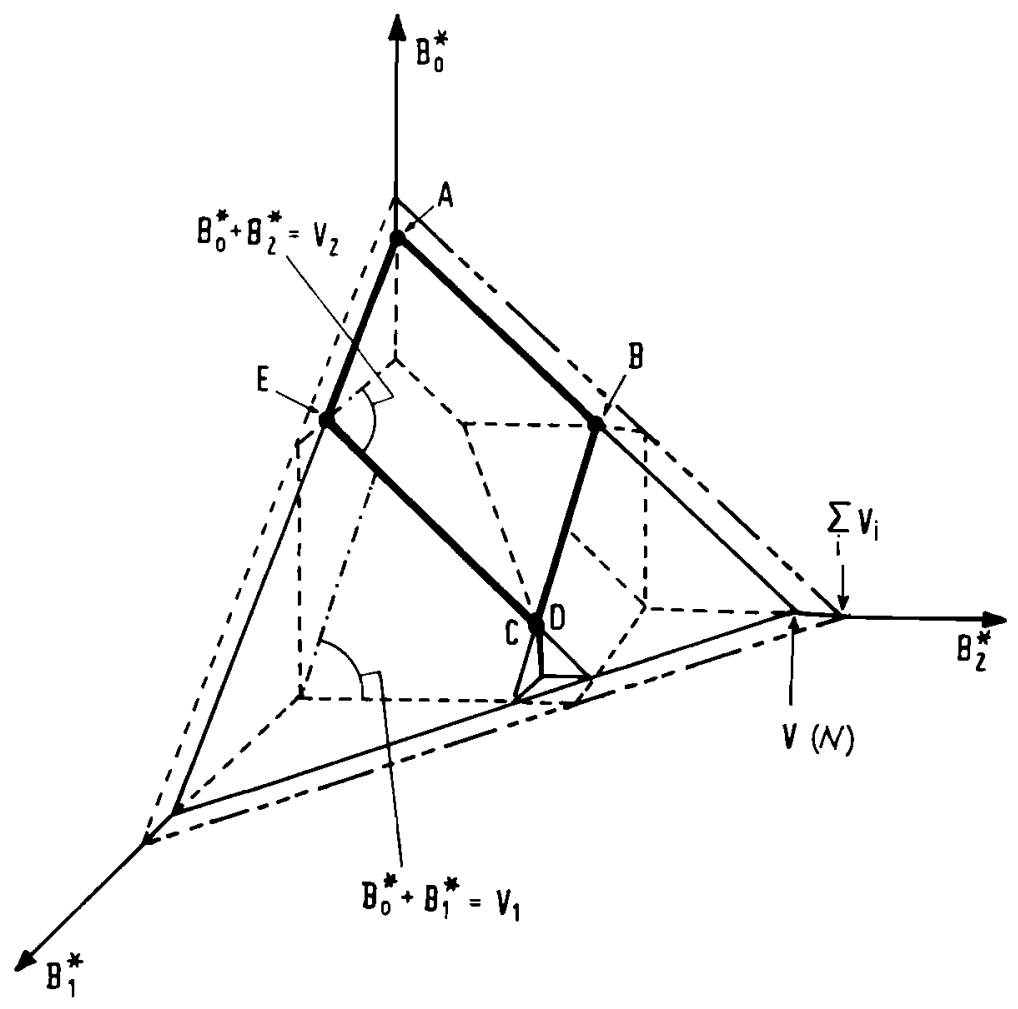

Figure 9. The core when $\mathrm{E}(\mathrm{q}) \neq 0$ and a completely disaggregated network is used.

The analysis of this situation can be accomplished in a way similar to the preceding ones and the main result is that the freedom to reallocate the rights of discharge gives rise to larger cores, i.e. the number of efficient taxation schemes is generally larger than in the case in which the C.A. cannot reallocate the rights of discharge.

Fixed Percentage of Removal and Taxes on $Q_{i}$

This time we assume that the law states that at least a certain percentage of each firm's waste production must be removed, i.e. if a firm produces $Q_{i}$ then the corresponding discharge must 
be $q_{i} \leq \bar{\alpha}_{i}$. As in the preceding case $A$, the Central Authority taking care of the waste treatment will select that effluent load vector $q^{\circ}$ that minimizes his cost. Again $q^{\circ}$ is in general a function of $Q$, while when the environmental damage is neglected we have $q_{+}^{0}=\bar{\alpha} Q_{+}$and $q_{i}^{0}=\bar{\alpha}_{i}$ respectively for the cases of aggregated and disaggregated treatment networks. The possible cases we consider are the same as before. Since the analysis can be developed in exactly the same way, we do not give any proof and we only summarize the main results.

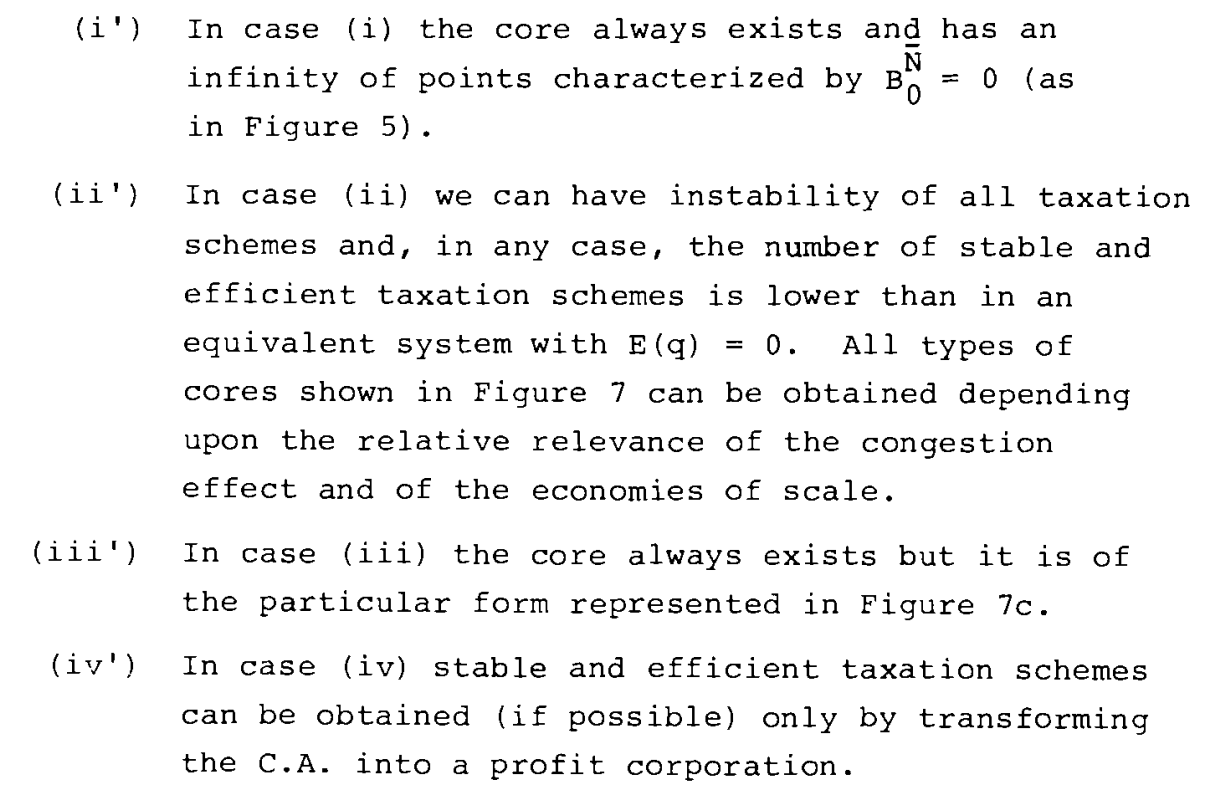

\section{Effluent Charges}

Let us suppose that the waste productions $\left\{\bar{Q}_{i}\right\}$ of the firms are given and assume that each firm treats its own waste. Thus, the cost of the C.A. is simply the cost of the environmental damage $E(q)$ and the charge is set on the effluents $q_{i}$. The demand function $A_{i}\left(q_{i}\right)$ is therefore given by

$$
A_{i}\left(q_{i}\right)=D_{i}\left(\bar{Q}_{i}\right)-T_{i}\left(\bar{Q}_{i}, q_{i}\right)
$$


and it is convex since $T_{i}\left(\bar{Q}_{i}, q_{i}\right)$ is convex (see property (a) of the cost of a treatment plant). This fact implies (see Remark 1) that efficient schemes can be generated.

Again the characteristic function cannot be proved to be convex and indeed by means of simple examples it could be shown that there are cases in which all efficient taxation schemes are unstable. More precisely the core exists only if the profits of the firms are sufficiently high with respect to the corresponding environmental damages. In any case the core, if it exists, is of the kind shown in Figure 9. Again the conclusion is that it is not possible to have stable taxation schemes with the C.A. being a non-profit corporation. (Stability has its price!)

The analysis of the case in which the waste production $Q_{i}$ of the firms has not yet been decided on and a standard on the percentage of removal is imposed can also be carried out. Proofs very similar to the ones we have al ready given are not reported here for the sake of brevity and the main result is that stable taxation schemes cannot be found if the profits of the firms are not sufficiently high. Moreover all stable taxation schemes are characterized by some positive benefit for the C.A.

\section{INTERPRETATION AND IMPLICATIONS OF THE RESULTS}

Very simple notions of the theory of games have been used in this paper to analyze the problems of stability and efficiency of taxation schemes in regional environmental management. Although the conceptualization presented in the paper applies to any kind of pollution problem (water, air, solid), reference is made to the problem of water pollution in a river basin and three particular classes of problems are analyzed in detail.

The first class is concerned with the case in which effluent standards are imposed by law on each firm. A regional authority is taking care of an areawide wastewater treatment system, the 
costs of which are apportioned among users by means of charges levied on the residuals produced by each firm. The existence of charging rules which allow the regional authority at least to cover its cost under the constraint that there is no incentive for any group of dischargers to withdraw from the regional system (stability) is analyzed. The role played by the damage to the environment and by the economics of scale in the treatment process are pointed out. If the environmental damage is negligible, stable taxation schemes can always be found, while, if the waste is treated in a completely disaggregated way (i.e., firm by firm), the opportunities for stable taxation schemes to exist are strongly limited.

The second class of problems is concerned with the case in which a standard on the percentage of removal is imposed by law to each firm (crude interpretation of the U.S. law (best practical treatment)). Again the taxes are on the waste production and results similar to those of the first case are found as far as the roles of the environmental damage and the structure of the network are concerned.

The third class of problems deals with the case of effluent charges. It is shown that stable taxation schemes can in general exist only if the firms are characterized by sufficiently high profits.

In conclusion, the paper shows that if the damages to the environment are not negligible and if these damages must be refunded by the users of the resource it is very unlikely that efficient and stable taxation schemes can be found if the regional authority acts as a non-profit corporation. These results also state that if the damages to the environment are neglected, or, in other words, if the total benefit of the firms instead of the social benefit is maximized, then efficiency and stability are easily obtained. This is indeed, what happened in the history of the industrial development of the last century in almost all countries: the efficiency of the firms has been very high and there has been no friction or competition for the use of the self- 
purification capacity of the environment. Nevertheless, these two nice attributes have been obtained at a price which is definitively too high: namely the fact that damages to society are neglected. The increased public awareness nowadays makes this solution no longer feasible. In this respect the paper indicates an alternative solution, since stability and efficiency can also be obtained by letting the regional authority get a profit by the sale of emission rights. The higher the environmental congestion the greater must be this profit. If ethical and political attitudes are against this kind of solution there is no way to maximize the social benefit without generating frictions among the polluters. 


\section{APPENDIX}

The proof technically consists in showing that the characteristic function $\mathrm{V}$ is convex, since then Property 3 can be applied. The proof is analogous to the one given by soreson et al. [7] for proving the convexity of $\mathrm{V}$ under slightly different conditions.

Consider two sets $A \subset B \subset \bar{N}$ and a firm $k \in \bar{N}-B$. For simplicity of notation let us denote

$$
A=A \cup\{\mathrm{k}\} \quad B=B \cup\{\mathrm{k}\}
$$

and recall that

$$
Q_{+}^{x}=\sum_{x} Q_{i}^{x} \quad \bar{q}_{+}^{x}=\sum_{x} \bar{q}_{i}
$$

where

$$
\mathrm{Q}_{\mathrm{i}}^{x}=0 \quad \forall \mathrm{i} \in x \quad \text { if } x=\underline{x} .
$$

Consider the two following alternative cases

$$
\begin{aligned}
& \text { (i) } Q_{+}^{A} \geq Q_{+}^{B} \\
& \text { (ii) } Q_{+}^{A}<Q_{+}^{B} .
\end{aligned}
$$

\section{Case (i)}

Equation (10) implies

$$
T\left(Q_{+}^{B}, \bar{q}_{+}^{B}\right)-T\left(\sum_{A} Q_{i}^{B}, \bar{q}_{+}^{A}\right) \geq T\left(Q_{+}^{A}+\sum_{B-A} Q_{i}^{B}, \bar{q}_{+}^{B}\right)-T\left(Q_{+}^{A}, \vec{q}_{+}^{A}\right)
$$

as it can be verified letting

$$
Q_{+}^{\prime}=\sum_{A} Q_{i}^{B} \quad q_{+}^{\prime}=\bar{q}_{+}^{A}
$$




$$
\begin{array}{rlrl}
Q_{+}^{\prime \prime} & =Q^{A} & q_{+}^{\prime \prime}=\bar{q}_{+}^{A} \\
\Delta=\sum_{B-A} Q_{i}^{B} & \delta=\bar{q}_{+}^{B-A} .
\end{array}
$$

Adding to both sides of Equation (A.1)

$$
V(A)-\sum_{A} A_{i}\left(Q_{i}^{B}\right)+T\left(Q_{+}^{A}, \bar{q}_{+}^{A}\right)
$$

and reordering, we obtain

$$
\begin{aligned}
& V(A)-\sum_{A} A_{i}\left(Q_{i}^{B}\right)+T\left(Q_{+}^{A}, \bar{q}_{+}^{A}\right)+T\left(Q_{+}^{B}, \bar{q}_{+}^{B}\right)-T\left(Q_{+}^{A}+\sum_{B-A} Q_{i}^{B}, \bar{q}_{+}^{B}\right) \\
& \geq V(A)-\sum_{A} A_{i}\left(Q_{i}^{B}\right)+T\left(\sum_{A} Q_{i}^{B}, \bar{q}_{+}^{A}\right) .
\end{aligned}
$$

Adding and subtracting $\sum_{B} A_{i}\left(Q_{i}^{B}\right)$ to the left hand side $H$ of the last inequality we get

$$
\sum_{A} A_{i}\left(Q_{i}^{A}\right)+\sum_{B-A} A_{i}\left(Q_{i}^{B}\right)-T\left(Q_{+}^{A}+\sum_{B-A} Q_{i}^{B}, \bar{q}_{+}^{B}\right)-V(B)=H .
$$

Noting that

$$
V(B) \geq \sum_{A} A_{i}\left(Q_{i}^{A}\right)+\sum_{B-A} A_{i}\left(Q_{i}^{B}\right)-T\left(Q_{+}^{A}+\sum_{B-A} Q_{i}^{B}, \vec{q}_{+}^{B}\right)
$$

we have

$$
\mathrm{V}(\mathrm{B})-\mathrm{V}(\mathrm{B}) \geq \mathrm{H} \text {. }
$$

On the other hand, since

$$
V(A) \geq \sum_{A} A_{i}\left(Q_{i}^{B}\right)-T\left(\sum_{A} Q_{i}^{B}, \bar{q}_{+}^{A}\right)
$$


we obtain

$$
V(A)-\sum_{A} A_{i}\left(Q_{i}^{B}\right)+T\left(\sum_{A} Q_{i}^{B}, \bar{q}_{+}^{A}\right) \geq V(A)-V(A)
$$

where the left hand side is the right hand side of Equation (A.2). Hence from (A.3) and (A.4) it follows

$$
V(B \cup\{k\})-V(B) \geq V(A \cup\{k\})-V(A) .
$$

\section{Case (ii)}

In this case Equation (10) implies

$$
T\left(Q_{+}^{A}, \bar{q}_{+}^{A}\right)-T\left(Q_{+}^{A}-Q_{k}^{A}, \bar{q}^{A}\right) \geq T\left(Q_{+}^{B}+Q_{k}^{A}, \bar{q}_{+}^{B}\right)-T\left(Q_{+}^{B}, \bar{q}_{+}^{B}\right)
$$

as can easily be proved.

Adding

$$
\sum_{A} A_{i}\left(Q_{i}^{A}\right)-\sum_{A} A_{i}\left(Q_{i}^{A}\right)-A_{k}\left(Q_{k}^{A}\right)
$$

and

$$
\sum_{B} A_{i}\left(Q_{i}^{B}\right)-\sum_{B} A_{i}\left(Q_{i}^{B}\right)-A_{k}\left(Q_{k}^{A}\right)
$$

(notice that the two expressions are equal) to the left and right hand sides respectively of Equation (A.5), and reordering, we get

$$
\begin{gathered}
{\left[\sum_{B} A_{i}\left(Q_{i}^{B}\right)+A_{k}\left(Q_{k}^{A_{1}}\right)-T\left(Q^{B}+Q_{k}^{A}, \bar{q}_{+}^{B}\right]-V(B)\right.} \\
\geq V(A)-\left[\sum_{A} A_{i}\left(Q_{i}^{A}\right)-T\left(Q_{+}^{A}-Q_{k}^{A}, \bar{q}^{A}\right)\right] .
\end{gathered}
$$

Finally, since 


$$
\begin{aligned}
& V(B) \geq \sum_{B} A_{i}\left(Q_{i}^{B}\right)+A_{k}\left(Q_{k}^{A}\right)-T\left(Q_{+}^{B}+Q_{k}^{A}, \bar{q}_{+}^{B}\right), \\
& V(A) \geq \sum_{A} A_{i}\left(Q_{i}^{A}\right)-T\left(Q_{+}^{A}-Q_{k}^{A}, \bar{q}_{+}^{A}\right),
\end{aligned}
$$

we obtain

$$
V(B \cup\{k\})-V(B) \geq V(A \cup\{k\})-V(A) \text {. }
$$




\section{LIST OF SYMBOLS}

$A_{i}\left(x_{i}\right)=$ demand function of the $i$ th polluter.

$A_{y}\left(x^{y}\right)=$ aggregated demand function for the subset $y$ of $N$.

$B_{i}\left(x_{i}\right)=A_{i}\left(x_{i}\right)-C_{i}\left(x_{i}\right)=$ benefit of the $i$ th polluter.

${ }^{B}{ }_{0}(x)=\sum_{N} C_{i}\left(x_{i}\right)=$ benefit of the C.A.

$B_{i}^{*} \quad=$ optimal benefit of the ith polluter.

$\mathrm{B}^{y} \quad=$ optimal benefit of the subset $y$ of $N$ in the case in which only the polluters of $\underline{y}$ are present in the system.

$c_{i}\left(x_{i}\right)=$ tax (charge) levied on the $i$ th polluter.

$C(x)=$ cost function of the C.A.

$c_{y}\left(x^{y}\right)=$ cost supported by the C.A. in the case in which only the polluters of the set $\underline{y}$ are present in the system.

$D_{i}\left(Q_{i}\right)=$ profit function of the ith firm.

$E(q)=$ environmental damage.

$N=$ the set of polluters.

$\bar{N} \quad=$ the set of polluters and the C.A.

$\mathrm{Q}_{\mathrm{i}} \quad=$ mass flow rate of pollutant produced by the ith firm.

$\bar{Q}_{i} \quad=$ a fixed level of waste production.

$Q_{+} \quad=\sum_{N} Q_{i}=$ total waste production.

Q = input vector of the treatment network.

$q_{j}=$ mass flow rate of pollutant discharged by the jth effluent of the network of treatment plants.

$q_{+} \quad=\sum_{j} q_{j}=$ total discharge.

$\vec{q}_{i} \quad=$ right of discharge of the ith firm.

$\vec{q}_{+} \quad=\sum_{N} \bar{q}_{i}=$ right of discharge of the C.A.

q = discharge vector of the treatment network.

$\mathrm{q}^{0}\left(Q_{+}\right)=$discharge vector of the least costly treatment network which treats the input $Q_{+}$with a total discharge $q_{+} \leq \bar{q}_{+}$. 


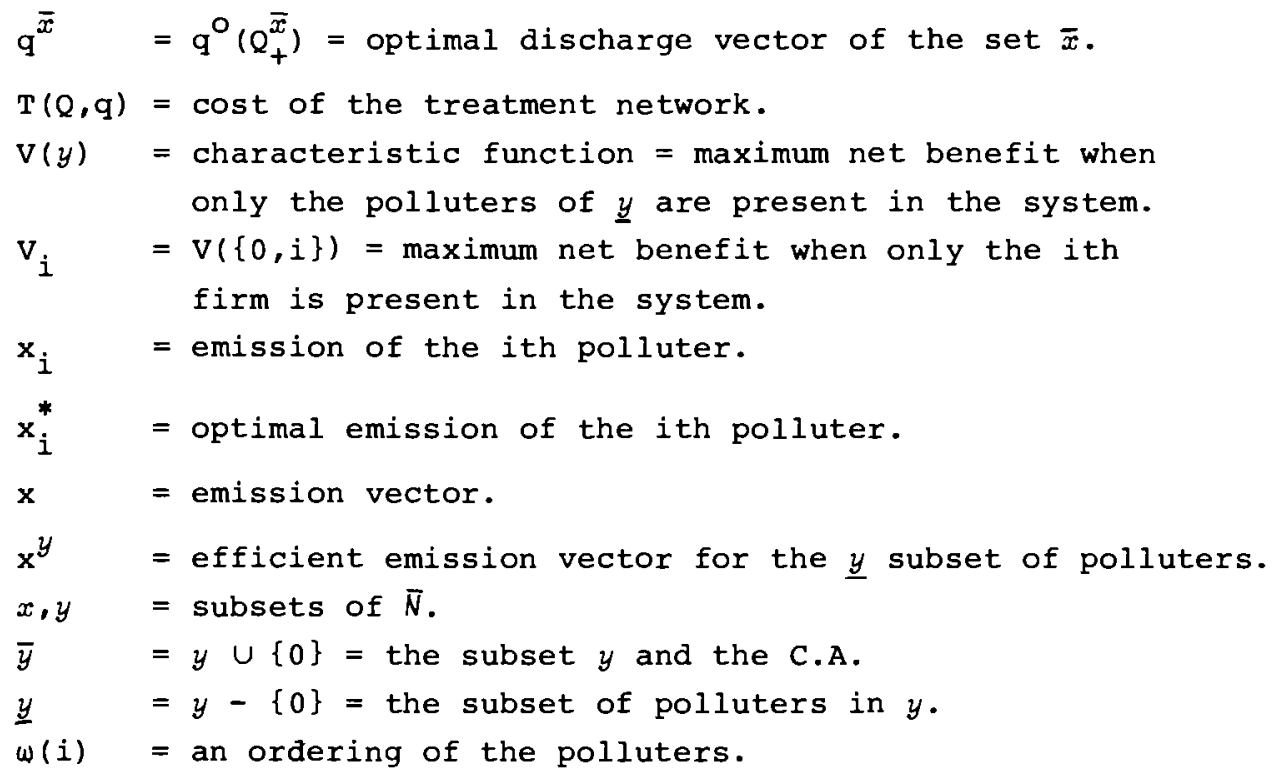




\section{REFERENCES}

[1] Upton, C., Optimal Taxing of Water Pollution, Water Resources Res., 4, 5 (1968), 865-875.

[2] Hass, J.E., Optimal Taxing for the Abatement of Water Pollution, Water Resources Res., 6, 2 (1970), 353-365.

[3] Ferrar, T.A., Progressive Taxation as a Policy for Water Quality Management, Water Resources Res., 9, 3 (1973), 563-568.

[4] Shapley, L.S., Cores of Convex Games, Int. J. of Game Theory, 1, 1 (1971), 11-26.

[5] Kneese, A.V., and B.T. Bower, Managing Water Quality: Economics, Technology, Institutions, Johns Hopkins Press, Baltimore, 1968.

[6] Kneese, A.V., Strategies for Environmental Management, Public Policy, 19 (1971), 37-52.

[7] Soreson, J., T. Tschirhart, and A.B. Whinston, A Theory of Pricing under Decreasing Costs. H.C. Krannert Graduate School of Industrial Administration, Purdue University, Lafayette, Indiana (to be published). 